\title{
Closed entry technique for the laparoscopic management of adnexal mass during pregnancy
}

\author{
Alejandro Correa-Paris • Elena Suárez-Salvador • \\ Antonia Gomar Crespo • Oriol Puig Puig • \\ Jordi Xercavins • Antonio Gil-Moreno
}

Received: 21 March 2014 / Accepted: 19 May 2014 / Published online: 15 June 2014

(C) Springer-Verlag Berlin Heidelberg 2014

\begin{abstract}
Management of adnexal masses during pregnancy is challenging for most gynecologists. When surgery is needed, a minimally invasive approach should be preferred. The aim of this study was to evaluate the safety and feasibility of the closed entry technique for laparoscopic management of adnexal masses during pregnancy. We reviewed clinical records and videos of laparoscopic procedures performed during pregnancy. Seventeen pregnant patients with diagnosis of adnexal mass that required surgery underwent laparoscopic surgery using the closed entry or Veress technique. We searched for complications related to surgery and obstetrical and perinatal outcomes. Median gestational age at the moment of surgery was 17 weeks (range, 6-30 $-30^{+4}$ weeks). A total of 18 interventions were performed: 12 salpingo-oophorectomies, 3 cystectomies, 1 salpingectomy, and 2 ovarian detorsions. There were no major operative or entry-related complications. Median hospital stay was 2 days (range, 1-5). Perinatal outcomes were as follows: four preterm births (all of them induced), nine full-term deliveries, one early pregnancy loss at 7 weeks, one miscarriage at 18 weeks, and two ongoing uncomplicated pregnancies. Laparoscopic approach using closed entry technique with an individual selection of the puncture site is safe in the management of adnexal masses that require surgery during pregnancy. In our experience, the Veress technique is more versatile as it gives the surgeon more
\end{abstract}

Electronic supplementary material The online version of this article (doi:10.1007/s10397-014-0851-7) contains supplementary material, which is available to authorized users.

A. Correa-Paris $(\bowtie) \cdot$ E. Suárez-Salvador • A. Gomar Crespo •

O. Puig Puig $\cdot$ J. Xercavins $\cdot$ A. Gil-Moreno

Servei de Ginecologia, Unitat de Ginecologia Oncològica, Hospital

Universitari Vall d'Hebron, Universitat Autònoma de Barcelona,

Passeig de la Vall d'Hebron 119-129, Barcelona 08035, Spain

e-mail: correa.alejandro@gmail.com

A. Correa-Paris

e-mail: acorrea@vhebron.net freedom to choose the location of the first trocar in patients with important space limitations due to the size of the adnexal mass and/or the enlarged gravid uterus.

Keywords Laparoscopic surgery · Pregnancy · Adnexal mass $\cdot$ Closed entry technique $\cdot$ Veress needle technique

\section{Background}

In the past years, there has been an increase in the diagnosis of adnexal pathology during pregnancy. This is in part due to the advances in assisted reproduction techniques that achieve pregnancies in older women with higher risk and also due to the routine use of ultrasound during gestation.

Adnexal masses are found in 2-10\% of pregnant patients; the incidence ranges between 1 and 81 in every 8,000 pregnancies. Incidence is higher in the first trimester and declines progressively throughout gestation. Malignancy is found in 1$10 \%$ of cases and is more frequent with increasing age [1-3]. The incidence reported in the literature includes the following differential diagnostics: dermoid cyst $(25 \%)$, corpus luteum cyst or functional cyst $(17 \%)$, serous cystadenoma $(14 \%)$, mucinous cystadenoma (11\%), endometriotic cyst (8\%), carcinoma (2.8\%), borderline tumor (3\%), and leiomyoma (2\%) [2].

Like in nonpregnant patients, suspicion of malignancy is based on ultrasound findings and individual clinical characteristics, for this the International Ovarian Tumor Analysis (IOTA) group criteria, and the use of an ultrasound-based risk of malignancy index (RMI) scoring system has been proven to be useful [4]. Traditional tumor markers for ovarian cancer are unreliable since they are physiologically elevated and vary according to gestational age. Spontaneous regression has been observed in 55-96\% of masses during gestation. Larger or more complex masses as well as those diagnosed later in gestation are more likely to persist. 
The most frequent complication is ovarian torsion, which appears in $7-15 \%$ of cases. Some studies have found a higher rate of torsion in the first trimester and for masses larger than $5 \mathrm{~cm} \mathrm{[5].}$

Generally, surgery is only indicated in patients with adnexal masses suspicious of malignancy, persistent or enlarging (higher risk of torsion), ruptured cysts, or symptomatic cases that do not respond to medical treatment. Although traditionally, laparotomy was used in pregnant patients requiring surgery, multiple studies have demonstrated the safety and feasibility of laparoscopy during gestation [6-9]. Advantages of laparoscopy over laparotomy have been well established in the general population: reduced postoperative pain and use of analgesia, less adhesions and postoperative ileus, lower infection rate, early return to activities and less thromboembolic risk, shorter hospital stay, and lower costs. In parallel, all of these benefits have also been shown in pregnant patients [10].

In the present study, we present our experience in the laparoscopic management of adnexal masses during pregnancy using the closed entry (Veress) technique and determine its safety and convenience, evaluating maternal and fetal complications as well as perinatal outcomes.

\section{Methods}

A retrospective study was carried out in the Vall d'Hebron University Hospital, between January 2008 and January 2014. Data was collected by reviewing clinical records and videos of all laparoscopic procedures performed during pregnancy in the first, second, or third trimester. Inclusion criteria were as follows: all operative laparoscopies using the closed entry or Veress technique, scheduled or emergency, indicated by the presence of an adnexal mass, suspicious of malignancy on ultrasound, or associated with abdominal pain unresponsive to medical therapy, suspicion of torsion, or whose characteristics did not allow for clinical follow-up. Exclusion criteria were laparotomy or laparoscopy using the open technique (Hasson). All patients had an ultrasound (US) prior to surgery. When malignancy was suspected, the US was performed by a specialist with experience in gynecological ultrasonography. Each patient's individual risk was determined using the IOTA criteria and personal risk factors (i.e., age, family history of ovarian or breast cancer, history of malignancy or endometriosis). We analyzed the following variables: patient's age, gestational age at diagnosis and at the moment of surgery, findings on ultrasound, type of surgery, surgical time (from initial incision to wound closure), and postoperative histopathologic diagnosis. We searched for complications associated with the entry technique and intervention, including abdominal organ injury (bowel or bladder), vascular injury, and febrile and infectious complications (e.g., postoperative fever, wound infection). We also recorded any complication that occurred throughout the gestation as well as obstetrical and perinatal outcomes. An ultrasound and/or fetal monitoring was made before and immediately after the operation to verify fetal well-being. No institutional review board approval was required at our hospital, given the characteristics of our study (chart review).

Abdominal access was achieved in all cases using the closed entry technique according to the classical procedure using the Veress needle (Lagis, Brussels) technique. On occasion, we alternatively used a Tuohy epidural needle (Smiths Medical, UK) as an alternative to the Veress needle. This type of needle is shorter ( $80 \mathrm{vs.} 120 \mathrm{~mm}$ ) and smaller in diameter (18 vs. $14 \mathrm{G})$. It is slightly curved at the end and has a blunted tip and little sharpness (Fig. 1).

The location for needle placement was chosen in the operating room by the surgeon according to patient's characteristics (e.g., previous surgery), uterine height, and size and the location of the mass (Fig. 2). All data were collected and analyzed in an Excel spreadsheet (Microsoft Corp., Redmond, WA, USA). We finally carried out a univariate statistical analysis for data presentation.

\section{Findings}

A total of 17 patients were included. The patients' median age was 34 years (range, 23-39 years). Median gestational age at diagnosis and at the moment of surgery was 12 weeks of gestation (range, $0-30^{+4}$ weeks) and 17 weeks (range, $6-30^{+4}$ weeks), respectively.

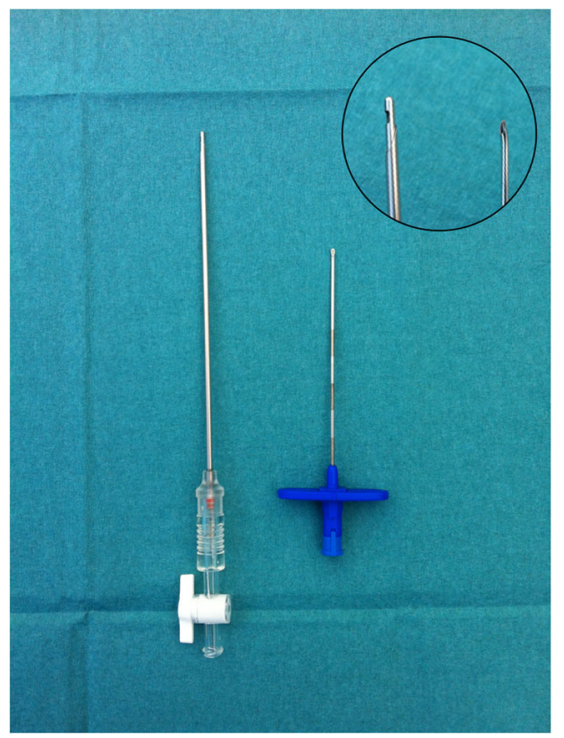

Fig. 1 Veress needle vs. Tuohy needle. Note the smaller dimensions of the epidural needle (right), being shorter and thinner than the Veress needle (left). A magnified image of each needle tip can be seen in the top right corner, showing the blunted tip of the Tuohy needle 


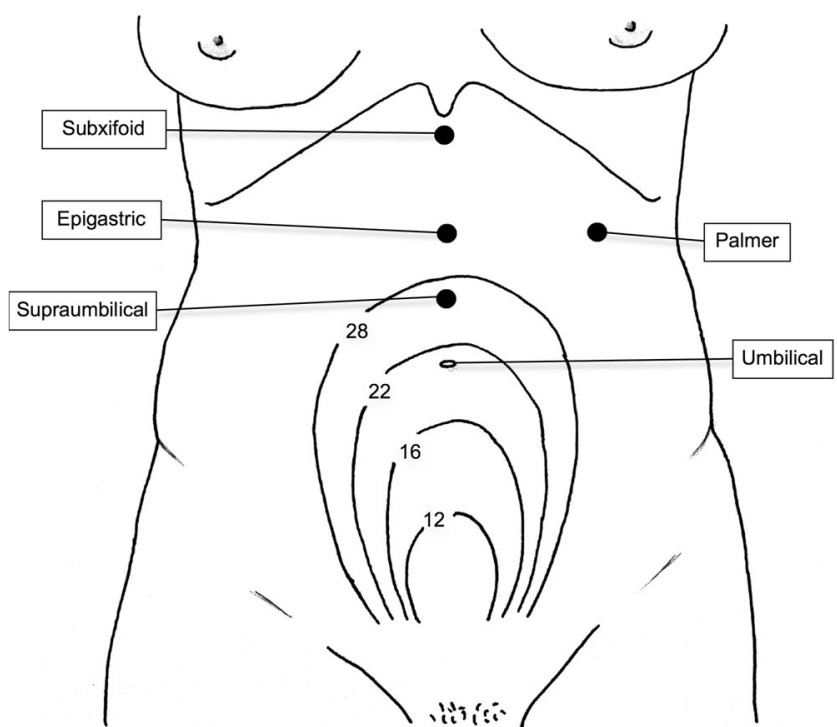

Fig. 2 Insertion sites of Veress needle according to gestational age and uterus size. The location for inserting the needle was determined by each surgeon in the operating room. Numbers indicate gestational age in weeks

Emergency surgery was necessary in six cases because of ovarian torsion. All cases are summarized in Table 1 including patient characteristics, complications, and obstetrical outcomes. Hospital stay was a median of 2 days (range, $1-5$ days). The number of trocars used was decided in the operating room according to each case. Two to four trocars were used, with diameters of 5,10 , and $11 \mathrm{~mm}$. Median surgical time was $60 \mathrm{~min}$ (range, 40-150 min). Only two surgeries required $150 \mathrm{~min}$ to be completed (cases nos. 2 and 10), one of them included peritoneal biopsies, and the other was an emergency laparoscopy under suspicion of ovarian torsion.

There were no conversions to laparotomy. No entry-related complications were observed. Only one case (no. 15) was complicated by postoperative anemia that required transfusion. This was the only twin pregnancy recorded in the cohort. An emergency laparoscopy was performed because of an ovarian torsion at $26^{+1}$ weeks, and difficult hemostasis because of venous bleeding from the ovarian pedicle was eventually achieved; the patient received a blood transfusion because of postoperative hemoglobin of $8 \mathrm{mg} / \mathrm{dL}$. She was otherwise clinically stable and the rest of her postoperatory was uneventful.

Tocolytics were not routinely used. As for obstetrical outcomes, we recorded one dilation and curettage, four cesarean and ten vaginal deliveries (including one breech delivery), and two ongoing uncomplicated pregnancies, with a total of 18 healthy newborns (including one twin pregnancy). All cesarean deliveries were done because of obstetrical indications (two for acute fetal distress, one for severe preeclampsia in a twin pregnancy, and one because of umbilical cord prolapse). There were no perinatal complications. In four patients, labor was induced preterm, three of them for obstetrical reasons and one for interval oncologic surgery in a patient with clear cell carcinoma who underwent neoadjuvant chemotherapy during pregnancy.

Gestational age at the moment of delivery was a median of 39 weeks (range, $18^{+2}-41$ weeks). We observed two fetal demises in cases where emergency surgery was performed. One of them occurred almost 10 weeks after surgery, presenting with oligohydramnios at 18 weeks and ending by vaginal delivery of a $170-\mathrm{g}$ fetus, following diagnosis of chorioamnionitis. The other was a miscarriage at 7 weeks of gestation after an emergency laparoscopy was performed 1 week earlier because of increasing pain and suspicion of heterotopic pregnancy (ultimately ruled out). Birth weights in the cohort were normal, with a median of 3,035 $\mathrm{g}$ (range, 170$3,870 \mathrm{~g})$.

Histopathologic analysis reported two cases of malignancy: one borderline ovarian serous tumor and an ovarian clear cell carcinoma. All of them were treated according to our oncology protocols. The case of clear cell carcinoma underwent neoadjuvant chemotherapy beginning at 18 weeks of pregnancy and until the 34th week when labor was induced for interval surgery (14 days postpartum). Optimal surgery was achieved laparoscopically with complete surgical staging. All biopsies were negative except for the peritoneal lavage cytology. This patient continued chemotherapy with no evidence of disease for 9 months after surgery. Ultimately, she presented with peritoneal carcinomatosis resistant to platinum chemotherapy. The patient died of complications of advancing disease 26 months after the initial diagnostic surgery. As for the borderline serous tumor case, pathology confirmed a FIGO stage IA, grade 2 tumor, therefore not requiring any additional procedure or surgical staging according to our protocols. This patient continued controls every 6 months at our center with negative tumoral markers and is presently without evidence of disease.

\section{Discussion}

When facing an adnexal mass, as in nonpregnant patients, the most feared outcome is malignancy. The risk of malignancy during pregnancy is approximately $2-3 \%[11-13]$; however, most cases are diagnosed in early stages and usually have good maternal and fetal prognosis [14].

In the context of gestation, other important risks are a higher rate of torsion (associated with the enlarged mass and gravid uterus), rupture of the mass, or mechanical obstruction of labor. Some authors recommend follow-up of adnexal masses diagnosed during gestation, especially in asymptomatic patients diagnosed during a routine prenatal ultrasound 
Table 1 Case description and outcomes of laparoscopy using closed entry (Veress) technique for adnexal masses during pregnancy

\begin{tabular}{|c|c|c|c|c|c|c|c|}
\hline Case & US findings ${ }^{\mathrm{a}}$ & $\begin{array}{l}\text { GE at } \\
\text { surgery }\end{array}$ & Intervention & Entry site & $\begin{array}{l}\text { Histopathologic } \\
\text { Diagnosis }\end{array}$ & Complications & Outcomes \\
\hline 1 & Complex mass ${ }^{\mathrm{b}}, 56 \mathrm{~mm}$ & $21^{+0}$ & $\mathrm{RSO}+$ peritoneal biopsy & SubX & $\begin{array}{l}\text { Serous micropapillary } \\
\text { borderline tumor }\end{array}$ & No & VD, 40 weeks, $3,870 \mathrm{~g}$ \\
\hline 2 & Complex mass ${ }^{\mathrm{b}}, 175 \mathrm{~mm}$ & $16^{+3}$ & LSO + peritoneal biopsy & EpiG & Bilateral clear cell carcinoma & No & ABD, 34 weeks, $2,280 \mathrm{~g}$ \\
\hline 3 & Simple cyst, $80 \mathrm{~mm}$ & $16^{+1}$ & RSO & $P$ & Mucinous cystadenoma & $\begin{array}{l}\text { PROM }+ \text { TPTL } \\
\text { at } 33^{+1} \text { weeks }\end{array}$ & $\mathrm{VD}, 33^{+5}$ weeks, $1,870 \mathrm{~g}$ \\
\hline 4 & Teratoma, $102 \mathrm{~mm}$ & $14^{+1}$ & LSO & $P$ & Mature cystic teratoma & No & $\mathrm{VD}, 39^{+1}$ weeks, $2,830 \mathrm{~g}$ \\
\hline 5 & Teratoma, $60 \mathrm{~mm}$ & $22^{+0}$ & $\mathrm{RSO}$ & SubX & Mature cystic teratoma & No & $\mathrm{CS}, 39^{+4}$ weeks, $3,100 \mathrm{~g}$ \\
\hline 6 & Complex mass ${ }^{\mathrm{b}}, 48 \mathrm{~mm}$ & $19^{+6}$ & Bilateral cystectomy & EpiG & Bilateral serous cystadenoma & No & VD, 39 weeks \\
\hline 7 & Simple cyst, $63 \mathrm{~mm}$ & $8^{+4}$ & Unilateral cystectomy & Umb & Endometriotic cyst & $\begin{array}{l}\text { Chorioamnionitis, } \\
\text { fetal demise }\end{array}$ & $\mathrm{VD}, 18^{+3}$ weeks, $170 \mathrm{~g}$ \\
\hline 8 & Teratoma, $74 \mathrm{~mm}$ & $20^{+1}$ & $\mathrm{RSO}$ & $\mathrm{P}$ & Mature cystic teratoma & No & $\mathrm{VD}, 39^{+1}$ weeks, $3,300 \mathrm{~g}$ \\
\hline 9 & Complex mass ${ }^{\mathrm{b}}, 126 \mathrm{~mm}$ & $13^{+5}$ & RSO & $\mathrm{P}$ & $\begin{array}{l}\text { Mucinous } \\
\text { cystadenoma }+ \text { torsion }\end{array}$ & No & $\mathrm{CS}, 40$ weeks, $3,700 \mathrm{~g}$ \\
\hline 10 & Ovarian torsion, $55 \mathrm{~mm}$ & $11^{+3}$ & LSO & Umb & Necrosis (torsion) & No & VD, 36 weeks, $3,100 \mathrm{~g}$ \\
\hline 11 & Simple cyst, $100 \mathrm{~mm}$ & $17^{+0}$ & LSO & SU & Endometriotic cyst & No & $\mathrm{VD}, 38$ weeks, $3,260 \mathrm{~g}$ \\
\hline 12 & Heterotopic pregnancy, $65 \mathrm{~mm}$ & $6^{+0}$ & Cystectomy + detorsion & $\mathrm{Umb}$ & Corpus luteum & Miscarriage & Miscarriage \\
\hline 13 & Adnexal torsion, $27 \mathrm{~mm}$ & $30^{+5}$ & R salpingectomy & EpiG & Necrosis (torsion) & No & CS, 41 weeks, $3,815 \mathrm{~g}$ \\
\hline 14 & $\begin{array}{l}\text { Ovarian tumor } \\
\quad \text { (possibly malignant), } 110 \mathrm{~mm}\end{array}$ & $20^{+1}$ & RSO & $\mathrm{P}$ & Mature cystic teratoma & No & Ongoing gestation \\
\hline 15 & Ovarian torsion, $68 \mathrm{~mm}$ & $26^{+1}$ & LSO & EpiG & Necrosis (torsion) & Blood transfusion & $\begin{array}{l}\mathrm{CS}, 34^{+3} \text { weeks, } 1,740 \\
\text { and } 1,540 \mathrm{~g}^{\mathrm{c}}\end{array}$ \\
\hline 16 & Simple cyst, $65 \mathrm{~mm}$ & $13^{+3}$ & Ovarian detorsion & $\mathrm{P}$ & - & No & Ongoing pregnancy \\
\hline 17 & Ovarian torsion, $73 \mathrm{~mm}$ & $19^{+2}$ & $\mathrm{RSO}$ & $\mathrm{P}$ & Necrosis (torsion) & No & Ongoing pregnancy \\
\hline
\end{tabular}

$G E$ gestational age given in weeks + days, $S u b X$ subxiphoid, EpiG epigastric, $P$ Palmer's point, $U m b$ umbilical, $S U$ supraumbilical, $R / L S O$ right/left salpingo-oophorectomy, TPTL threatened preterm labor, $V D$ vaginal delivery, $A B D$ assisted breech delivery, $C S$ cesarean section

${ }^{\text {a }}$ Ultrasound (US) findings describe the mass characteristics or suspected diagnosis based on US and the maximum diameter of the mass (or in cases of ovarian torsion, maximal ovarian diameter)

${ }^{\mathrm{b}}$ Refers to presence of septations and/or papillary projections with US suspicion of ovarian carcinoma

${ }^{\mathrm{c}} \mathrm{CS}$ delivery because of severe preeclampsia in a twin dichorionic diamniotic pregnancy

where a high percentage of masses found in the first trimester may spontaneously resolve.

Although torsion or rupture may be rare, there are no data regarding the ultrasonographic characteristics that could predict such risk. The risk of torsion appears to be greater in masses between 6 and $10 \mathrm{~cm}$ [5]. Surgical management is necessary for those masses suspicious of malignancy, at high risk of torsion, or symptomatic and nonresponsive to medical therapy.

Although laparoscopic management of adnexal masses during pregnancy is safe and feasible (when surgical management is warranted), most obstetricians/gynecologists are still reluctant and only well-trained and experienced endoscopic surgeons are at ease with this approach. An additional theoretical advantage of laparoscopy during gestation is the reduced manipulation of the uterus, which could minimize the potential risk of preterm birth.

In our hospital, over 10,000 routine prenatal ultrasound studies (first and second trimester) were performed between the study period; we found an incidence of adnexal mass of
$1.1 \%$, similar to other published studies. Our practice results are also consistent with other series in rate of malignancy with an incidence of $1.7 \%$ in pregnant patients (unpublished data). According to previously exposed criteria, only $20 \%$ of pregnant patients with persistent adnexal mass underwent surgery in our hospital (unpublished results). In the present cohort, the elevated rate of malignancy (2/17) is due to a careful selection of patients that do require surgery.

Koo et al. have published the results of laparoscopic surgical management of 11 cases of adnexal complex benign masses during the first trimester of gestation. In their study, all laparoscopies were emergency operations, and they did not encounter any miscarriages and surgical or obstetric complications [15]. Another retrospective study described the results of 11 patients undergoing laparoscopy for adnexal mass in the second trimester also showing favorable outcomes and no complications [16].

In our present cohort, we performed elective surgery during the second and third trimester of pregnancy. The only five patients that underwent laparoscopy in the first trimester were 
emergency surgeries. The two fetal losses observed occurred after emergency surgery, one miscarriage after emergent laparoscopy performed at 6 weeks of gestation, and a late abortion due to chorioamnionitis 10 weeks after an emergency laparoscopy was performed at 8 weeks.

Approximately $50 \%$ of complications during laparoscopic surgery are entry-related. This proportion has not changed in the last 20 years. According to recent guidelines for laparoscopy during pregnancy published by the Society of American Gastrointestinal and Endoscopic Surgeons (SAGES), entry can be performed with an open (Hasson) technique, Veress needle, or optical trocar, if the location is adjusted according to fundal height and previous incisions [17]. However, there is still much concern for the blind entry with the Veress needle because of its potential complications, namely, injury to the gravid uterus [18], hence the ongoing debate regarding abdominal access in the pregnant patient.

So far, no significant or clinically relevant differences have been found between any technique [19-22]. A meta-analysis published recently found no difference in major vascular or visceral complications. There was, however, a lower incidence of failed entry with the open technique, as well as less extraperitoneal insufflation and omental lesion [23].

As we lack enough evidence or even a unified consensus, the surgeon's experience and familiarization with any given technique are still more important. Most studies published regarding management of adnexal masses during gestation encourage the use of open or Hasson technique [19, 20, 22, 24], speculating that there is a higher risk of injury to the gravid uterus using the Veress needle method. Some studies have been published using the closed entry (Veress) technique $[15,16,25]$, although there are no trials comparing the two in pregnant patients.

Because of the normal anatomical variations in pregnancy and the reduction of space due to the enlarged gravid uterus and adnexal mass, we must change our strategy for insertion of the trocars and insufflation of pneumoperitoneum. We believe that the closed entry technique permits more freedom in choosing the trocar insertion sites, since it allows a more accurate evaluation of the patient's abdomen once the $\mathrm{CO}_{2}$ has been insufflated. Also, we evaluate the port site for presence of adhesions using Palmer's safety test and our "safety cone" technique (see video). With abdominal distention caused by pneumoperitoneum, it is easier to determine the best location for insertion of the first trocar according to the size of the gravid uterus and both size and location of the adnexal mass (Fig. 3). This is not possible with the open technique since the site chosen for insufflation will inevitably be the location of the first trocar (Hasson-type trocar), which usually is large in diameter $(\geq 10 \mathrm{~mm})$. Using the Veress needle technique also helps to reduce the use of large-diameter trocars to reposition the endoscope and therefore the possible complications associated with the use of such trocars, particularly the potentially

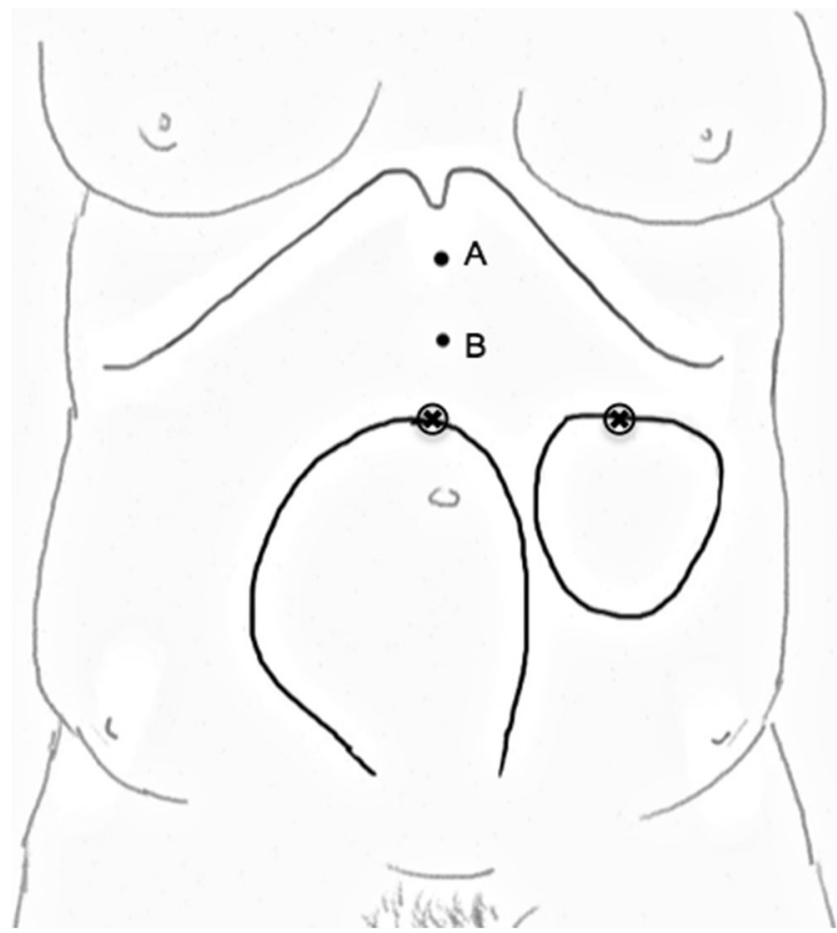

Fig. 3 Case of a left adnexal mass in a patient with a 17 -week pregnancy. Abdominal entry at the supraumbilical or Palmer's point is not possible due to high risk of injury ( $X$ marks). Alternative entry points can be the subxiphoid $(A)$ or epigastric $(B)$ locations

increased risk of trocar site hernia with the use of these large trocars in pregnant patients.

In our present cohort, the use of the closed entry (Veress) technique proved to be safe and no complications occurred. It was never necessary to change the main trocar to reposition the endoscope. We also believe that the use of the Tuohy needle has the same advantages as the Veress needle, which are enhanced by its smaller dimensions, allowing for less potential risk of injury to the gravid uterus.

\section{Conclusions}

In summary, our findings support the safety of operative laparoscopy using the closed entry (Veress) technique in pregnant patients with adnexal masses diagnosed during pregnancy, with satisfactory maternal and perinatal outcomes. When managing adnexal masses in pregnant patients, we must take into account the risk of neoplasia and the risk of associated complications such as torsion, rupture, or obstetrical issues associated with such masses. Also, we must consider the risk of surgery at an early gestational age during the first trimester and the potential risks of emergency surgery with possibly worse obstetrical outcomes.

We believe that a closed entry technique using the Veress needle — or a similar needle (i.e., Tuohy needle)—is safe, useful, and convenient since it allows more freedom in 
choosing the location for inserting the first trocar in these specific cases. More large prospective trials are needed to establish if there is any difference between the open and closed technique for operative laparoscopy in pregnant patients diagnosed with adnexal mass.

Conflict of interest On behalf of all authors, the corresponding author states that there is no conflict of interest.

Informed consent This article does not contain any studies with human or animal subjects performed by the any of the authors. Therefore, no additional informed consent was required by our institution's Ethics Committee.

\section{References}

1. Schwartz N, Timor-Tritsch IE, Wang E (2009) Adnexal masses in pregnancy. Clin Obstet Gynecol 52:570-585. doi:10.1097/GRF. 0b013e3181bea9d7

2. Hoover K, Jenkins TR (2011) Evaluation and management of adnexal mass in pregnancy. Am J Obstet Gynecol 205:97-102. doi:10. 1016/j.ajog.2011.01.050

3. Yazbek J, Helmy S, Ben-Nagi J et al (2007) Value of preoperative ultrasound examination in the selection of women with adnexal masses for laparoscopic surgery. Ultrasound Obstet Gynecol 30: 883-888. doi:10.1002/uog.5169

4. Timmerman D, Ameye L, Fischerova D et al (2010) Simple ultrasound rules to distinguish between benign and malignant adnexal masses before surgery: prospective validation by IOTA group. BMJ 341:c6839

5. Lee GSR, Hur SY, Shin JC et al (2004) Elective vs. conservative management of ovarian tumors in pregnancy. Int J Gynaecol Obstet 85:250-254. doi:10.1016/j.ijgo.2003.12.008

6. Reedy MB, Källén B, Kuehl TJ (1997) Laparoscopy during pregnancy: a study of five fetal outcome parameters with use of the Swedish Health Registry. Am J Obstet Gynecol 177:673-679

7. Oelsner G, Stockheim D, Soriano D et al (2003) Pregnancy outcome after laparoscopy or laparotomy in pregnancy. J Am Assoc Gynecol Laparosc 10:200-204

8. Koo Y-J, KIM HJ, Lim K-T et al (2011) Laparotomy versus laparoscopy for the treatment of adnexal masses during pregnancy. Aust N Z J Obstet Gynaecol 52:34-38. doi:10.1111/j.1479-828X.2011.01380.x

9. Soriano D, Yefet Y, Seidman DS et al (1999) Laparoscopy versus laparotomy in the management of adnexal masses during pregnancy. Fertil Steril 71:955-960
10. Lee Y-Y, Kim T-J, Choi CH et al (2010) Factors influencing the choice of laparoscopy or laparotomy in pregnant women with presumptive benign ovarian tumors. Int J Gynaecol Obstet 108:12-15. doi:10.1016/j.ijgo.2009.07.040

11. Bernhard LM, Klebba PK, Gray DL, Mutch DG (1999) Predictors of persistence of adnexal masses in pregnancy. Obstet Gynecol 93:585589

12. Whitecar MP, Turner S, Higby MK (1999) Adnexal masses in pregnancy: a review of 130 cases undergoing surgical management. Am J Obstet Gynecol 181:19-24

13. Ueda M, Ueki M (1996) Ovarian tumors associated with pregnancy. Int J Gynaecol Obstet 55:59-65

14. Leiserowitz GS, Xing G, Cress R et al (2006) Adnexal masses in pregnancy: how often are they malignant? Gynecol Oncol 101:315321. doi:10.1016/j.ygyno.2005.10.022

15. Ko ML, Lai TH, Chen SC (2009) Laparoscopic management of complicated adnexal masses in the first trimester of pregnancy. Fertil Steril 92:283-287. doi:10.1016/j.fertnstert.2008.04.035

16. Stepp KJ, Tulikangas PK, Goldberg JM et al (2003) Laparoscopy for adnexal masses in the second trimester of pregnancy. J Am Assoc Gynecol Laparosc 10:55-59

17. Pearl J, Price R, Richardson W, Fanelli R (2011) Guidelines for diagnosis, treatment, and use of laparoscopy for surgical problems during pregnancy. Surg Endosc 25:3479-3492. doi:10.1007/s00464011-1927-3

18. Friedman JD, Ramsey PS, Ramin KD, Berry C (2002) Pneumoamnion and pregnancy loss after second-trimester laparoscopic surgery. Obstet Gynecol 99:512-513

19. Yuen PM, Ng PS, Leung PL, Rogers MS (2004) Outcome in laparoscopic management of persistent adnexal mass during the second trimester of pregnancy. Surg Endosc 18:1354-1357. doi:10.1007/ s00464-003-8283-x

20. Mathevet P, Nessah K, Dargent D, Mellier G (2003) Laparoscopic management of adnexal masses in pregnancy: a case series. Eur $\mathrm{J}$ Obstet Gynecol Reprod Biol 108:217-222

21. Moore RD, Smith WG (1999) Laparoscopic management of adnexal masses in pregnant women. J Reprod Med 44:97-100

22. Balthazar U, Steiner AZ, Boggess JF, Gehrig PA (2011) Management of a persistent adnexal mass in pregnancy: what is the ideal surgical approach? J Minim Invasive Gynecol 18:720-725. doi:10.1016/j. jmig.2011.07.002

23. Ahmad G, O'Flynn H, Duffy JMN et al (2012) Laparoscopic entry techniques. Cochrane Database Syst Rev 2, CD006583. doi:10.1002/ 14651858.CD006583.pub3

24. Rizzo AG (2003) Laparoscopic surgery in pregnancy: long-term follow-up. J Laparoendosc Adv Surg Tech A 13:11-15. doi:10. 1089/109264203321235403

25. Koo Y-J, Lee J-E, Lim K-T et al (2011) A 10-year experience of laparoscopic surgery for adnexal masses during pregnancy. Int $\mathrm{J}$ Gynaecol Obstet 113:36-39. doi:10.1016/j.ijgo.2010.10.020 